\title{
Ossifying fibroma vs fibrous dysplasia of the jaw: molecular and immunological characterization
}

Satoru Toyosawa ${ }^{1}$, Michiko Yuki ${ }^{1}$, Mitsunobu Kishino ${ }^{1}$, Yuzo Ogawa ${ }^{1}$, Takafumi Ueda ${ }^{2}$, Shumei Murakami ${ }^{3}$, Eiichi Konishi ${ }^{4}$, Seiji Iida ${ }^{5}$, Mikihiko Kogo ${ }^{5}$, Toshihisa Komori ${ }^{6}$ and Yasuhiko Tomita ${ }^{7}$

${ }^{1}$ Department of Oral Pathology, Osaka University Graduate School of Dentistry, Suita, Osaka, Japan;

${ }^{2}$ Department of Orthopedic Surgery, National Hospital Organization, Osaka National Hospital, Osaka, Japan;

${ }^{3}$ Department of Oral and Maxillofacial Radiology, Osaka University Graduate School of Dentistry, Suita, Osaka, Japan; ${ }^{4}$ Department of Pathology, Kyoto Prefecture General Hospital, Kyoto, Japan; ${ }^{5}$ Department of Oral Surgery, Osaka University Graduate School of Dentistry, Suita, Osaka, Japan; ${ }^{6}$ Department of Developmental and Reconstructive Medicine, Nagasaki University Graduate School of Biomedical Sciences, Nagasaki, Japan and ${ }^{7}$ Department of Pathology, Osaka University Graduate School of Medicine, Suita, Osaka, Japan

Ossifying fibroma and fibrous dysplasia of the jaw are maxillofacial fibro-osseous lesions that should be distinguished each other by a pathologist because they show distinct patterns of disease progression. However, both lesions often show similar histological and radiological features, making distinction between the two a diagnostic dilemma. In this study, we performed immunological and molecular analyses of five ossifying fibromas, four cases of extragnathic fibrous dysplasia, and five cases of gnathic fibrous dysplasia with typical histological and radiographic features. First, we examined the difference between fibrous dysplasia and ossifying fibroma in the expression of Runx2 (which determined osteogenic differentiation from mesenchymal stem cells) and other osteogenic markers. Fibroblastic cells in fibrous dysplasia and ossifying fibroma showed strong Runx2 expression in the nucleus. The bone matrices of both lesions showed similar expression patterns for all markers tested except for osteocalcin. Immunoreactivity for osteocalcin was strong throughout calcified regions in fibrous dysplasia, but weak in ossifying fibroma lesions. Second, we performed PCR analysis with peptide nucleic acid (PNA) for mutations at the $\mathrm{Arg}^{201}$ codon of the alpha subunit of the stimulatory G protein gene (GNAS), which has reported to be a marker for extragnathic fibrous dysplasia. All nine cases of extragnathic or gnathic fibrous dysplasia were positive for this mutation. On the other hand, none of the five cases of ossifying fibroma showed the mutation. These findings indicate that although fibrous dysplasia and ossifying fibroma are similar disease entities, especially in the demonstration of the osteogenic lineage in stromal fibroblast-like cells, they show distinct differences that can be revealed by immunohistochemical detection of osteocalcin expression. Furthermore, PCR analysis with PNA for GNAS mutations at the Arg ${ }^{201}$ codon is a useful method to differentiate between fibrous dysplasia and ossifying fibroma.

Modern Pathology (2007) 20, 389-396. doi:10.1038/modpathol.3800753

Keywords: fibrous dysplasia; ossifying fibroma; differential diagnosis; GNAS mutation; Runx2; bone matrix proteins

Maxillofacial fibro-osseous lesions comprise a group of face and jaw disorders characterized by the replacement of bone by a benign connective-tissue

Correspondence: Dr S Toyosawa, DDS, PhD, Department of Oral Pathology Osaka University Graduate School of Dentistry, 389-396 Yamadaoka, Suita, Osaka 565-0871, Japan.

E-mail: toyosawa@dent.osaka-u.ac.jp

Received 13 November 2006; revised 21 December 2006; accepted 29 December 2006 matrix with varying amounts of mineralized substances. Ossifying fibroma and fibrous dysplasia are the most common fibro-osseous lesions, which may be associated with significant cosmetic and functional disturbances; as they show distinct patterns of disease progression, it is important to distinguish between the two. Because of its risk for recurrence, ossifying fibroma needs to be completely enucleated from the surrounding bone. ${ }^{1-3}$ In contrast, patients with fibrous dysplasia should be 
treated according to their clinical presentation. Monostotic fibrous dysplasia growth usually tends to stabilize when skeletal maturity is attained; therefore, surgical intervention in children and adolescents should be delayed as long as possible. ${ }^{1-4}$ For patients with symptomatic fibrous dysplasia, bisphosphonate therapy is utilized..$^{5,6}$ Typical cases of ossifying fibroma and fibrous dysplasia of face and jaw are distinguished by radiological and histological appearances. However, these lesions often present a diagnostic dilemma because of uncertainties concerning the diagnostic significance of specific radiological and histological features; therefore, accurate diagnosis of these lesions can be difficult. $^{1-3}$

Histologically, stromal fibroblasts produce bony matrix without morphologic evidence of osteoblastic cells at the periphery of the bony spicules in fibrous dysplasia. ${ }^{4}$ Ultrastructural and biochemical studies have suggested that the fibroblastic component of fibrous dysplasia is related to the osteogenic lineage $;^{7,8}$ however, precise molecular biological evidence for this has not yet been presented. Recently, it was demonstrated that osteogenic differentiation from mesenchymal stem cells is controlled by the transcriptional factor Runx2..$^{9,10}$ Therefore, differences in the expression of Runx2 and other osteogenic markers might permit the histological distinction between the fibrous dysplasia and ossifying fibroma.

An activating point mutation of the alpha subunit of the stimulatory $\mathrm{G}$ protein gene (GNAS) at the Arg $^{201}$ codon was identified in lesional tissues of patients with McCune-Albright syndrome. ${ }^{11}$ Subsequently, GNAS mutations were observed in extragnathic fibrous dysplasias without McCuneAlbright syndrome, ${ }^{12-15}$ recognizing it as a marker of fibrous dysplasia. However, the presence or absence of GNAS mutations in gnathic fibrous dysplasias has not been examined.
In the present study, we analyzed the expression of osteogenic markers and GNAS mutations in cases of gnathic fibrous dysplasia and ossifying fibroma with typical radiological and histological features, in order to identify important markers that permit differentiation between these two disease entities.

\section{Materials and methods}

\section{Experimental Subjects}

Nine specimens of fibrous dysplasia, five of ossifying fibroma, two of osteofibrous dysplasia and three of normal bones were decalcified in EDTA solution, fixed in formalin, and embedded in paraffin. Tissue samples used in this study were obtained in compliance with the guidelines of the Joint Commission on Clinical Investigation of Osaka University Graduate School of Dentistry and Medicine. The nine fibrous dysplasias comprised five monostotic gnathic cases, and three-monostotic and one-polyostotic extragnathic cases. All five cases of ossifying fibroma were derived from the gnathic area. These lesions were all diagnosed as typical cases based on the clinical, radiological and histological criteria. The clinical data associated with these cases are summarized briefly in Table 1 . Histological sections of these samples cut at $5 \mu \mathrm{m}$ were stained with hematoxylin and eosin and subjected to immunoperoxidase procedures.

\section{Immunohistochemistry}

Immunohistochemical staining was performed by the streptavidin-biotin complex (sABC) peroxidase method with the anti-mouse or anti-rabbit sABC system from Dako (Glostrup, Denmark). The primary antibodies used in the present study were as follows: mouse anti-Runx2 monoclonal antibody (MBL Co, Ltd, Nagoya, Japan), ${ }^{16}$ rabbit anti-dentin

Table 1 Clinical data of the patients and results of GNAS mutational analysis

\begin{tabular}{|c|c|c|c|c|}
\hline Patient & Age/sex & Diagnosis & Site & GNAS mutation \\
\hline 1 & $37 / \mathrm{F}$ & Monostotic fibrous dysplasia & Femur & R201 H \\
\hline 2 & $38 / \mathrm{F}$ & Monostotic fibrous dysplasia & Femur & R201 H \\
\hline 3 & $25 / \mathrm{F}$ & Polyostotic fibrous dysplasia & Femur & R201 C \\
\hline 4 & $31 / \mathrm{F}$ & Monostotic fibrous dysplasia & Femur & R201 C \\
\hline 5 & $69 / \mathrm{F}$ & Monostotic fibrous dysplasia & Maxilla & R201 H \\
\hline 6 & $55 / \mathrm{M}$ & Monostotic fibrous dysplasia & Maxilla & R201 H \\
\hline 7 & $15 / F$ & Monostotic fibrous dysplasia & Maxilla & R201 C \\
\hline 8 & $11 / \mathrm{F}$ & Monostotic fibrous dysplasia & Mandible & R201 C \\
\hline 9 & $18 / \mathrm{F}$ & Monostotic fibrous dysplasia & Maxilla & R201 C \\
\hline 10 & $49 / \mathrm{M}$ & Ossifying fibroma & Mandible & None \\
\hline 11 & $14 / \mathrm{M}$ & Ossifying fibroma & Maxilla & None \\
\hline 12 & $13 / \mathrm{M}$ & Ossifying fibroma & Maxilla & None \\
\hline 13 & $31 / \mathrm{F}$ & Ossifying fibroma & Maxilla & None \\
\hline 14 & $24 / \mathrm{F}$ & Ossifying fibroma & Mandible & None \\
\hline
\end{tabular}

R201 H, Arg to His; R201C, Arg to Cys; None, no mutation. 
matrix protein 1 (DMP1) polyclonal antibody, ${ }^{17}$ mouse anti-bovine osteocalcin monoclonal antibody (clone; OC4-3) (TaKaRa Biomedicals, Shiga, Japan), and rabbit anti-mouse osteopontin polyclonal antibody (IBL Co, Ltd, Gunma, Japan). Antigen retrieval was performed by trypsin digestion for the immunostaining of osteocalcin and DMP1, and heating with citrate buffer for the immunostaining of Runx2 and osteopontin. Sections were lightly counterstained with methyl green. As negative controls, mouse or rabbit IgG sera (Dako) were used as primary antibodies, giving uniformly negative results.

\section{Polymerase Chain Reaction-Restriction Fragment Length Polymorphism (PCR-RFLP)}

Genomic DNA was extracted from paraffin-embedded tissue blocks using DNeasy Tissue Kit (QIAGEN, Tokyo, Japan). Fifty nanograms of genomic DNA was used for PCR amplification with Pfu Ultra ${ }^{\mathrm{TM}}$ Hotstart High-Fidelity DNA polymerase (Stratagene, La Jolla, CA, USA) and primers P1 (5'-CCATTGACCTCAATTTTGTTT CAG-3') and P2 (5'-GGTAACAGTTGGCTTACTG GAAGTTG-3' $)^{18,19}$ for 30 cycles. The PCR-amplified products were diluted 20 times in distilled water and used for nested PCR with mutant primers P3 (5'-AGGACCTG CTTCGCGGC- $3^{\prime}$ ) and P4 (5'-CAGTT GGCTTACTGGAAGTTGACT TT-3') for 30 cycles. To block the amplification of the wild-type allele, a peptide nucleic acid (PNA) primer (Gly-NH2-CGCTGCCG TGTC-HAc) (FASMAC, Co Ltd, Kanagawa, Japan) was added to the first and nested-PCR reactions. ${ }^{15,20}$ The P3 primer creates a new restriction site for EagI (CGGCCG) through the change to $G$ in the first position of codon 200 in the normal allele; therefore, the 88-bp fragment amplified by the nested PCR is divided into 74- and 14-bp fragments upon EagI digestion. On the other hand, the allele containing a GNAS mutation at the first or second position of the $\mathrm{Arg}^{201}$ codon is not digested by EagI. After EagI treatment, the PCR products were separated by $4 \%$ NuSieve GTG agarose gel electrophoresis (FMC BioProducts, Rockland, ME, USA) and stained with ethidium bromide to visualize the amplified bands.

\section{Sequencing}

The 88-bp amplified products of the nested PCR with a PNA primer, were purified by $2 \%$ agarose gel electrophoresis. The bands were excised and isolated from the gel by DNA extraction kits (QIAGEN). The isolated DNA was subcloned into the pGEM-T Easy vector (Promega Co, Madison, WI, USA). After purification, sequencing was carried out using vector primers SP6 and T7. Ten clones per a lesion were analyzed using an automated DNA sequencer model 373 (Applied Biosystems, Foster City, CA,
USA). Thus, all PCR-amplified products were sequenced to confirm the presence of the mutation.

\section{Results}

\section{Clinicopathological and Radiological Findings of the Patients}

Clinical data of the patients with fibrous dysplasia and ossifying fibroma are listed in Table 1. Nine fibrous dysplasias and five ossifying fibromas presented as enlargement of the affected bones, and five gnathic fibrous dysplasias and five ossifying fibromas showed facial asymmetry. Radiologically, gnathic fibrous dysplasia showed homogeneous radio-dense opacities with a ground-glass appearance that blends into the surrounding normal bone (Figure 1a), whereas ossifying fibroma appeared as a unilocular mixed radiolucent and radio-opaque lesion with sharply defined borders (Figure 1d). Histologically, gnathic fibrous dysplasia is composed of irregular-shaped woven bone within a vascularized fibrous stroma of variable cellularity (Figure $1 \mathrm{~b}$ and $\mathrm{c}$ ). The woven bone spicules are evenly distributed throughout the lesion and exhibit a variety of shapes (Figure $1 \mathrm{~b}$ and $\mathrm{c}$ ). Ossifying fibromas showed prominent calcified structures (ossicles and cementicles) that appeared as eosinophilic or basophilic spherules of osteoid or bone within a moderately cellular, dense stroma (Figure $1 \mathrm{e}$ and f). Thus, the lesions satisfied with their typical clinical, radiological, and histological criteria were used in this study.

\section{Immunohistochemical Findings of Fibrous Dysplasia and Ossifying Fibroma}

The immunohistochemical findings from the extragnathic and gnathic fibrous dysplasia and ossifying fibroma are presented in Figure 2. In both fibrous dysplasia and ossifying fibroma, Runx2 staining was observed in the nuclei of spindle cells within fibrous connective tissues as well as cells on the surfaces of mineralized structures (Figure 2a-c). Likewise, osteopontin was detected around the periphery of calcified structures in both lesions (Figure 2d-f). Strong immunoreactivity against osteocalcin was distributed throughout calcified structures in fibrous dysplasia (Figure $2 \mathrm{~g}$ and $\mathrm{h}$ ), whereas only weak immunoreactivity was visible in ossifying fibroma (Figure 2i). DMP1 was specifically present in the calcified matrix surrounding osteocyte-like cells in both fibrous dysplasia and ossifying fibroma (Figure 2j-l). Thus, there was no significant difference between fibrous dysplasia and ossifying fibroma in the bone matrix distribution of any of the proteins tested except for osteocalcin. 

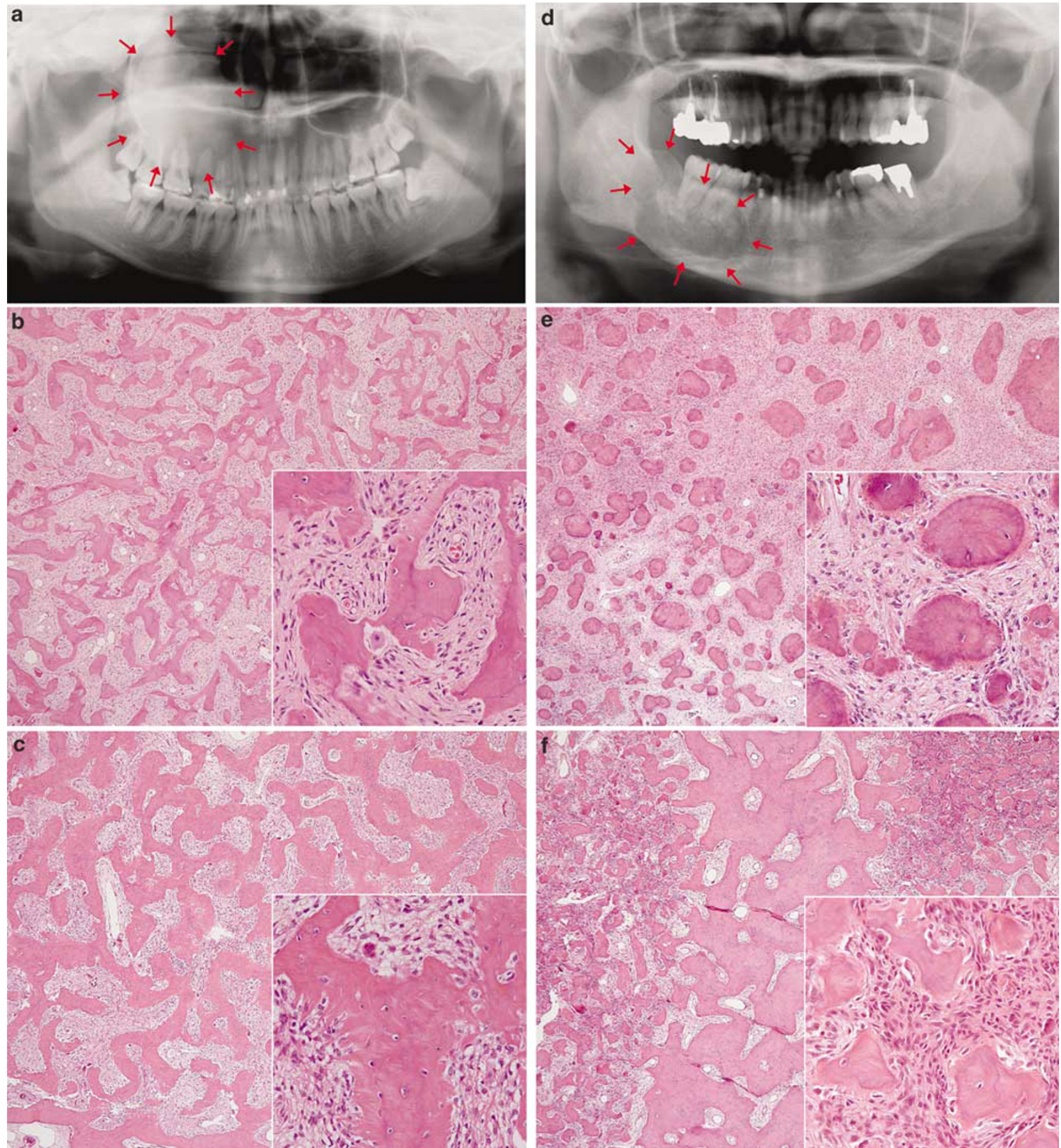

Figure 1 Representative panoramic radiographs (a, d) and photomicrographs of H\&E-stained (b, c, e, f) gnathic fibrous dysplasia (a-c) and ossifying fibroma (d-f). In panoramic radiographs, gnathic fibrous dysplasia is homogeneously radio-opaque with a ground-glass appearance and poorly defined margins indicated by the red arrow (a). In contrast, ossifying fibroma appears as a unilocular mixed radiolucent and radio-opaque lesion with well-defined borders, indicated by the red arrow (d). The photomicrographs show that gnathic fibrous dysplasia shows thin irregular-shaped woven bone that resembles membranous ossification (b, c). Ossifying fibroma has a moderately cellular, dense fibrous stroma with prominent calcified spherules corresponding to ossicles and cementicles (e, f). Original magnifications: (b), (c), (e), $(\mathbf{f}) \times 40$; (insets) $\times 400$.

\section{Detection of GNAS Mutation in Fibrous Dysplasia and Ossifying Fibroma Using PCR-RFLP}

DNA samples from normal bone (Figure 3a, lanes $1-3,7-9$ ) and osteofibrous dysplasia (Figure 3a, lanes $4,5,10,11)$ were used as negative controls. ${ }^{18}$ When PCR was performed without PNA, an 88-bp amplified fragment was generated in the negative controls. With the addition of PNA to the PCR, the intensity of the amplified bands was reduced by 



Figure 2 Representative immunohistochemical results from paraffin-embedded extragnathic fibrous dysplasia (a, d, g, j), gnathic fibrous dysplasia $(\mathbf{b}, \mathbf{e}, \mathbf{h}, \mathbf{k})$, and ossifying fibroma $(\mathbf{c}, \mathbf{f}, \mathbf{i}, \mathbf{l})$ stained with anti-Runx2 (a-c), anti-osteopontin (d-f), anti-osteocalcin (g-i), and antiDMP1 ( $\mathbf{j}-\mathbf{l}$ ) antibodies. Runx2 is detected in the nuclei of fibroblastic cells within fibrous tissue in extragnathic fibrous dysplasia, gnathic fibrous dysplasia, and ossifying fibroma (a-c). There was no significant difference in bone-matrix expression between fibrous dysplasia and ossifying fibroma of any proteins except for osteocalcin $(\mathbf{d}-\mathbf{f}$ and $\mathbf{j}-\mathbf{l})$. Extragnathic and gnathic fibrous dysplasias, however, showed strong immunoreactivity for osteocalcin in calcified materials ( $\mathbf{g}$ and $\mathbf{h}$ ), whereas ossifying fibroma stained only weakly for osteocalcin in comparable regions (i). Original magnifications: $(\mathbf{a}-\mathbf{l}) \times 200$. 

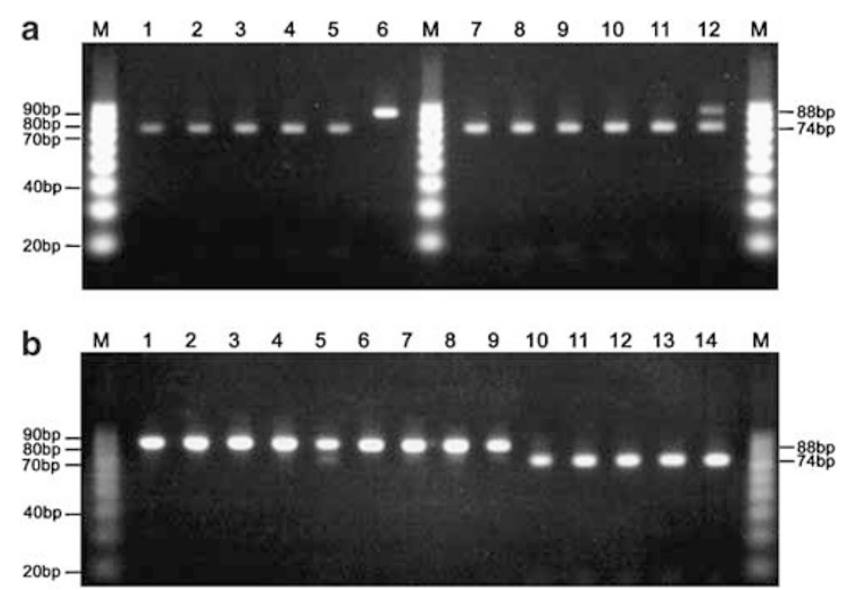

Figure 3 Mutational analysis of the GNAS gene at the $\mathrm{Arg}^{201}$ codon using PCR-RFLP. (a) Control PCR-RFLP experiments examining the GNAS gene at the $\mathrm{Arg}^{201}$ codon in the presence (lanes 1-6) or absence (lanes 7-12) of PNA. In the presence and absence of PNA, EagI digested the 88-bp amplified DNA fragments, including codon 201 of the GNAS gene into 74- and 14-bp fragments in normal bones (lanes 1-3 and 7-9) and osteofibrous dysplasias (lanes $4,5,10,11$ ) as negative controls. The 14-bp fragment was not visible in this gel. In contrast, polyostotic fibrous dysplasia showed undigested 88-bp fragments (lanes 6, 12). In the absence of PNA, undigested 88-bp (mutant allele) and digested 74-bp (normal allele) bands are visible (lane 12). In the presence of PNA, only the undigested 88-bp band is visible (lane 6). These results indicate that selective amplification of mutant alleles is performed in the presence of PNA. The 10-bp ladder was used as a size marker. (b) PCR-RFLP analysis of the $\mathrm{Arg}^{201}$ codon of the GNAS gene in extragnathic fibrous dysplasia (lanes 1-4), gnathic fibrous dysplasia (lanes 5-9), and ossifying fibroma (lanes 10-14) in the presence of PNA. After Eagl digestion, all extragnathic and gnathic fibrous dysplasias showed persistent undigested 88-bp fragments (lanes 1-9). In contrast, all ossifying fibromas showed digested 74-bp fragments (lanes 10-14). A 10-bp ladder was used as a size marker.

approximately $40-60 \%$. After EagI treatment, the amplified 88-bp fragments were completely digested into two fragments 74- and 14-bp in length, regardless of whether or not PNA was present (Figure 3a, lanes 1-5, 7-11). Further sequencing analysis revealed no GNAS mutation in these DNA samples (data not shown).

A DNA sample from a paraffin block of extragnathic polyostotic fibrous dysplasia (patient No. 3) was used as a positive control. In the absence of PNA, undigested 88-bp and digested 74-bp bands, corresponding to the mutant and wild-type alleles, respectively, were visible after EagI digestion (Figure 3a, lane 12). In the presence of PNA, only the undigested 88-bp band, and not the digested 74-bp band, was visible after EagI digestion (Figure 3a, lane 6). These results indicate the selective amplification of mutant alleles in the presence of PNA. ${ }^{20}$

PCR analysis was performed for four extragnathic fibrous dysplasias, five gnathic fibrous dysplasias, and five ossifying fibromas. In all nine cases of extragnathic or gnathic fibrous dysplasia, the amplified 88-bp fragments were not digested by EagI (Figure 3b, lanes 1-4: extragnathic fibrous dysplasia; 5-9: gnathic fibrous dysplasia). In contrast, EagI treatment of the five samples of ossifying fibroma resulted into complete digestion into the 74 - and 14bp fragments (Figure 3b, lanes 10-14). Sequencing analysis revealed GNAS mutations at the $\mathrm{Arg}^{201}$ codon in all nine cases of fibrous dysplasia, but in none of the ossifying fibroma samples (Table 1).

\section{Discussion}

Ossifying fibroma and fibrous dysplasia often present a diagnostic dilemma for both clinicians and pathologists because of their radiographic and histological similarity. Voytek et $a l^{21}$ reported that the considerable ossifying fibroma were completely indistinguishable from fibrous dysplasia from their histological studies. These lesions also demonstrated considerable radiographic overlap. ${ }^{21}$ They suggested that, because of this similarity, ossifying fibroma and fibrous dysplasia could be considered as diseases at either end of a single morphological spectrum. An additional report suggested that ossifying fibroma is a variant of fibrous dysplasia rather than a distinct disease entity. ${ }^{22}$ The present study clearly demonstrates that immunohistochemical analysis of osteocalcin and PCR analysis of GNAS mutations are useful methods in the differentiation between the two, and furthermore suggests that they are probably distinct disease entities.

Fibrous dysplasia is characterized histologically by stromal fibroblasts producing bony matrix without morphological evidence of osteoblastic cells at the periphery of the bony spicules. The osteoblastic nature of these fibroblastic cells has been suggested by electron microscopic analysis showing a lining of abnormal osteoblasts with a fibroblast-like appearance around the immature woven bone, ${ }^{7}$ and biochemical analysis showing an increase in alkaline phosphatase activity in the cells populating the fibrotic areas of fibrous dysplasia. ${ }^{8}$ In the present study, the nuclei of fibroblastic cells, as well as cells on the surface of bones, showed strong expression of Runx2, an important transcription factor for the osteogenic lineage, indicating that these cells are the osteogenic precursors. Similarly, the fibroblastic cells in ossifying fibroma also showed strong Runx2 expression in the nucleus. Both lesions could thus be interpreted as diseases of cells in the osteogenic lineage.

Immunohistochemical analysis for osteogenic markers such as osteopontin and DMP1 did not show much difference between fibrous dysplasia and ossifying fibroma; however, osteocalcin immunohistochemistry demonstrated a marked difference between the two. Osteopontin, osteocalcin, and DMP1 are abundant noncollagenous proteins of the normal bone matrix. ${ }^{23-25}$ Osteocalcin, the most abundant noncollagenous protein, ${ }^{24}$ is distributed throughout normal bone ${ }^{25}$ and has been shown by gene knockout technology to be a negative regulator of bone formation. ${ }^{26}$ The abundance of osteocalcin 
in fibrous dysplasia and its deficiency in ossifying fibroma suggests that the calcified material in fibrous dysplasia is more similar to normal bone than that in ossifying fibroma. This marked difference may indicate differences in bone formation and osteoblast differentiation between the two lesions.

Analysis of GNAS mutations at the $\operatorname{Arg}^{201}$ codon was found to be useful in distinguishing between ossifying fibroma and fibrous dysplasia. The somatic nature of the mutations in fibrous dysplasia makes their identification difficult, because the mutations are not present in all cells of affected patients, even within affected organs. Therefore, a PNA-clamping method was employed in the present study. ${ }^{15,20}$ Sequencing of the PCR-amplified fragments confirmed the accuracy of the PCR analysis using PNA, suggesting that the PCR analysis used in the present study may be a useful and easily available method for detecting GNAS mutations at the $\operatorname{Arg}^{201}$ codon.

Alterations in the tumor suppressor gene HPRT2 in ossifying fibroma have recently been reported. ${ }^{27}$ Direct sequencing of the HPRT2 revealed mutations in two out of the four cases of ossifying fibroma. ${ }^{27}$ These findings indicate that the HPRT2 mutation is not common in the development of ossifying fibroma, and therefore may not be used as a marker for diagnosis.

In conclusion, fibrous dysplasia and ossifying fibroma are similar disease entities in that both show markers consistent with the osteogenic lineage in their stromal fibroblast-like cells. They are distinct, however, in the precise composition of bone matrix, as shown by osteocalcin immunohistochemistry. Finally, PCR analysis with PNA for GNAS mutations at the $\mathrm{Arg}^{201}$ codon is a potentially useful method to differentiate between the two.

\section{Acknowledgement}

This work was supported by Grant-in-aid 17390484 and 18659539 from the Ministry of Education, Culture, Sports, Science and Technology of Japan, and the 21st Century COE Program.

\section{References}

1 Waldron CA. Fibro-osseous lesions of the jaws. J Oral Maxillofac Surg 1993;51:828-835.

2 Brannon RB, Fowler CB. Benign fibro-osseous lesions: a review of current concepts. Adv Anat Pathol 2001;8:126-143.

3 Alawi F. Benign fibro-osseous diseases of the maxillofacial bones. A review and differential diagnosis. Am J Clin Pathol 2002;118(Suppl):S50-S70.

4 Sciubba JJ, Fantasia JE, Kahn LB (eds). Fibro-osseous Lesions. AFIP Atlas of Tumor Pathology. Tumors and Cysts of the Jaw, 3rd Series, Fasc29 edn. Armed Forces Institute of Pathology: Washington, DC, 1999, pp 141-160.
5 Liens D, Delmas PD, Meunier PJ. Long-term effects of intravenous pamidronate in fibrous dysplasia of bone. Lancet 1994;343:953-954.

6 Kos M, Luczak K, Godzinski J, et al. Treatment of monostotic fibrous dysplasia with pamidronate. J Craniomaxillofac Surg 2004;32:10-15.

7 Greco MA, Steiner GC. Ultrastructure of fibrous dysplasia of bone: a study of its fibrous, osseous, and cartilaginous components. Ultrastruct Pathol 1986; 10:55-66.

8 Riminucci M, Fisher LW, Shenker A, et al. Fibrous dysplasia of bone in the McCune-Albright syndrome: abnormalities in bone formation. Am J Pathol 1997;151:1587-1600.

9 Komori T, Yagi H, Nomura S, et al. Targeted disruption of Cbfa1 results in a complete lack of bone formation owing to maturational arrest of osteoblasts. Cell 1997;89:755-764.

10 Otto F, Thornell AP, Crompton T, et al. Cbfa1, a candidate gene for cleidocranial dysplasia syndrome, is essential for osteoblast differentiation and bone development. Cell 1997;89:765-771.

11 Weinstein LS, Shenker A, Gejman PV, et al. Activating mutations of the stimulatory $G$ protein in the McCune-Albright syndrome. N Engl J Med 1991;325: 1688-1695.

12 Malchoff CD, Reardon G, MacGillivray DC, et al. An unusual presentation of McCune-Albright syndrome confirmed by an activating mutation of the Gs alphasubunit from a bone lesion. J Clin Endocrinol Metab 1994;78:803-806.

13 Shenker A, Weinstein LS, Sweet DE, et al. An activating Gs alpha mutation is present in fibrous dysplasia of bone in the McCune-Albright syndrome. J Clin Endocrinol Metab 1994;79:750-755.

14 Alman BA, Greel DA, Wolfe HJ. Activating mutations of Gs protein in monostotic fibrous lesions of bone. J Orthop Res 1996;14:311-315.

15 Bianco P, Riminucci M, Majolagbe A, et al. Mutations of the GNAS1 gene, stromal cell dysfunction, and osteomalacic changes in non-McCune-Albright fibrous dysplasia of bone. J Bone Miner Res 2000;15: 120-128.

16 Zhang YW, Yasui N, Ito K, et al. A RUNX2/PEBP2alpha A/CBFA1 mutation displaying impaired transactivation and Smad interaction in cleidocranial dysplasia. Proc Natl Acad Sci USA 2000;97:10549-10554.

17 Toyosawa S, Tomita Y, Kishino M, et al. Expression of dentin matrix protein 1 in tumors causing oncogenic osteomalacia. Mod Pathol 2004;17:573-578.

18 Candeliere GA, Roughley PJ, Glorieux FH. Polymerase chain reaction-based technique for the selective enrichment and analysis of mosaic $\arg ^{201}$ mutations in $\mathrm{G}$ alphas from patients with fibrous dysplasia of bone. Bone 1997;21:201-206.

19 Sakamoto A, Oda Y, Iwamoto Y, et al. A comparative study of fibrous dysplasia and osteofibrous dysplasia with regard to Gs alpha mutation at the $\mathrm{Arg}^{201}$ codon: polymerase chain reaction-restriction fragment length polymorphism analysis of paraffin-embedded tissues. J Mol Diagn 2000;2:67-72.

20 Lietman SA, Ding C, Levine MA. A highly sensitive polymerase chain reaction method detects activating mutations of the GNAS gene in peripheral blood cells in McCune-Albright syndrome or isolated fibrous dysplasia. J Bone Joint Surg Am 2005;87: 2489-2494. 
21 Voytek TM, Ro JY, Edeiken J, et al. Fibrous dysplasia and cemento-ossifying fibroma. A histologic spectrum. Am J Surg Pathol 1995;19:775-781.

22 Sissons HA, Steiner GC, Dorfman HD. Calcified spherules in fibro-osseous lesions of bone. Arch Pathol Lab Med 1993;117:284-290.

23 Mark MP, Prince CW, Oosawa T, et al. Immunohistochemical demonstration of a 44-KD phosphoprotein in developing rat bones. J Histochem Cytochem 1987;35:707-715.

24 Hauschka PV, Lian JB, Gallop PM. Direct identification of the calcium-binding amino acid, gamma-carboxy- glutamate, in mineralized tissue. Proc Natl Acad Sci USA 1975;72:3925-3929.

25 Toyosawa S, Shintani S, Fujiwara T, et al. Dentin matrix protein 1 is predominantly expressed in chicken and rat osteocytes but not in osteoblasts. J Bone Miner Res 2001;16:2017-2026.

26 Ducy P, Desbois C, Boyce B, et al. Increased bone formation in osteocalcin-deficient mice. Nature 1996; 382:448-452.

27 Pimenta FJ, Gontijo Silveira LF, et al. HRPT2 gene alterations in ossifying fibroma of Tavares GC, the jaws. Oral Oncol 2006;42:735-759. 